\title{
Numerical Prediction of Bubbly Flow Around an Oscillating Hydrofoil by Incompressible Two-Fluid Model
}

TOMOMI UCHIYAMA*

Center for Information Media Studies, Nagoya University, Furo-cho, Chikusa-ku, Nagoya 464-8603, Japan

This paper is concerned with the calculation of bubbly flow around a hydrofoil (NACA65-010), which is forced to oscillate normal to the uniform flow. An incompressible two-fluid model, expressed in the ALE (Arbitrary LagrangianEulerian) form, is solved by the finite element method using an upstream scheme. The flow field around the hydrofoil changes in accordance with the oscillation of the hydrofoil, and vortices are periodically shed from the trailing edge. The unsteady change in the forces acting on the hydrofoil is clarified, and the relation between the forces and the periodical flow around the hydrofoil is discussed.

Keywords: Numerical simulation; Gas-liquid two-phase flow; Turbopump; ALE finite element method; Unsteady force; Phase distribution

Related to the safety analysis of nuclear reactors and the development of crude oil pumps, attention has been given to the performance of turbopumps handling gas-liquid two-phase mixtures (Mikielewicz et al., 1978; Minemura \& Uchiyama, 1993). Most studies have been done on the pump performance under steady operating condition, in which the pump flow rate and rotational speed remain unaltered. Thus, the performance and the flow in the impeller under unsteady conditions, such as starting and stopping periods, have scarcely been investigated except in a few studies (Kastner \& Seeberger, 1983; Narabayashi et al., 1986).

With the intention of clarifying the unsteady performance of pumps operated under single-phase flow conditions, the flow around a hydrofoil, which is forced to oscillate normal

\footnotetext{
Received 15 September 2000; in final form 7 January 2001.

*Tel./Fax: +81-52-789-5187. E-mail: uchiyama@info.human.nagoyau.ac.jp
}

to the uniform flow, was studied. Ohashi and Ishikawa (1971) examined the effects of the oscillation frequency and amplitude on the wake of a hydrofoil using a flow visualization technique. Oshima (1982) calculated the flow by a discrete vortex method and compared the results with the corresponding measurements.

To grasp the flow mechanism of gas-liquid two-phase mixtures around a stationary hydrofoil, Ichikawa et al. (1991) measured the distribution of void fraction, and Shida et al. (1986) and Nishikawa et al. (1991) numerically analyzed the flow. But the two-phase flow around an oscillating hydrofoil has not yet been studied.

The author (Uchiyama, 2001) has proposed a finite element method for gas-liquid two-phase flow around a moving boundary. In the method, an incompressible twofluid model is expressed in the ALE (Arbitrary LagrangianEulerian) form, and the model is solved by the Galerkin finite element method. The author (Uchiyama, 2001) has applied the method to calculate the flow around a circular cylinder, which is forced to oscillate in a quiescent airwater two-phase mixture, and confirmed that the numerical result is favorably compared with the existing one under the single-phase condition.

In this study, the bubbly flow around a hydrofoil, which is forced to oscillate normal to the uniform flow, was simulated by the abovementioned ALE finite element method in conjunction with an upstream scheme (Uchiyama, 1999a, 1999b). The simulation made clear the unsteady change in the forces acting on the hydrofoil and revealed the relation between the forces and the periodical flow around the hydrofoil.

\section{PROBLEM DESCRIPTION}

The flow around a hydrofoil oscillating in an air--water bubbly flow on a horizontal plane is calculated. Figure 1 shows the hydrofoil and the computational domain. The 


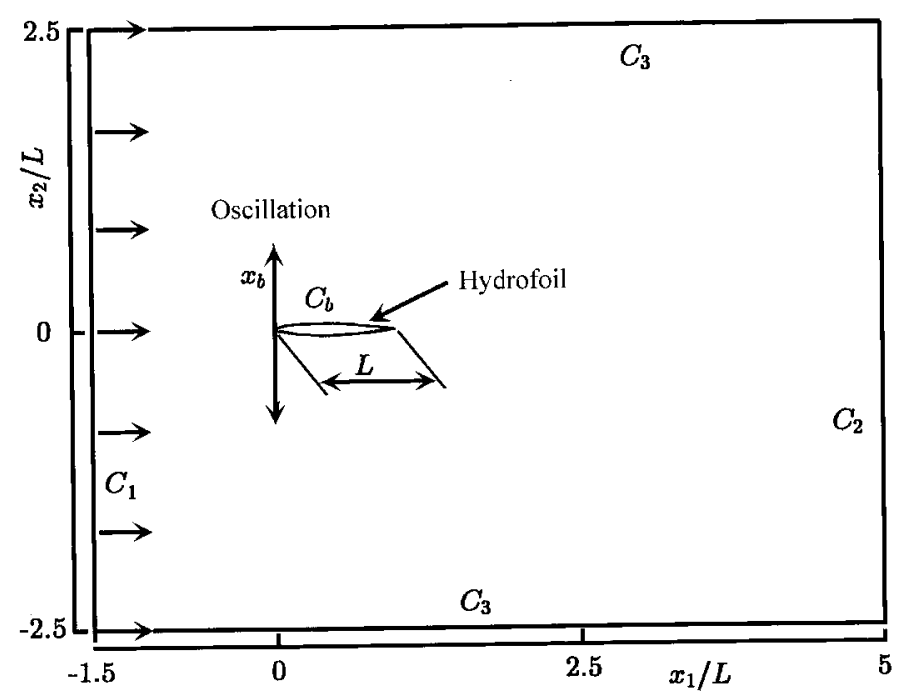

FIGURE 1 Oscillating hydrofoil and computational domain.

hydrofoil, NACA65-010, at an angle of attack of 0 degrees in the bubbly flow, is forced to oscillate sinuously normal to the uniform flow. The displacement of the hydrofoil $x_{b}$ is given by the following equation:

$$
x_{b} / L=(a / L) \sin \left(2 \pi t^{*}\right)
$$

where $a$ is the oscillation amplitude, $L$ is the chord length, and the dimensionless time $t^{*}$ is defined by using the oscillation period $T$ as $t^{*}=t / T$.

The calculation is made on a rectangular region of $6.5 L \times 5 L$, as shown in Figure 1 .

\section{BASIC EQUATIONS}

\section{Incompressible Two-Fluid Model}

It is postulated that both phases are incompressible and that the mixture flows isothermally without phase change. A one-pressure two-fluid model (Ishii, 1975) gives the conservation equations of the mass and momentum for each phase:

$$
\begin{gathered}
\frac{\partial \alpha_{k}}{\partial t}+\frac{\partial}{\partial x_{j}}\left(\alpha_{k} u_{k j}\right)=0 \quad(k=g, l) \\
\frac{\partial u_{k i}}{\partial t}+u_{k j} \frac{\partial u_{k i}}{\partial x_{j}}=-\frac{1}{\rho_{k}} \frac{\partial p}{\partial x_{i}}+\frac{1}{\alpha_{k} \rho_{k}} \frac{\partial}{\partial x_{j}}\left(\alpha_{k} \tau_{k i j}\right)+f_{k i} \\
(k=g, l)
\end{gathered}
$$

where

$$
\alpha_{g}+\alpha_{l}=1
$$

Subscript $k$ denotes the gas $(k=g)$ or liquid $(k=l)$ phase, and subscripts $k, g, l$ disobey the summation convention in this paper. In Eq. [3], $\tau_{k}$ is the viscous stress and $f_{k}$ is the interfacial forces.

As the forces $f_{k}$, an interfacial drag force $f_{k i}^{D}$, a virtual mass force $f_{k i}^{V}$, and a lift force $f_{k i}^{L}$ are taken into account (Uchiyama, 2000).

$$
\begin{gathered}
f_{k i}^{D}=-\Lambda_{k}\left(u_{g i}-u_{l i}\right) \quad(k=g, l) \\
f_{k i}^{V}=-\Gamma_{k}\left(\frac{\partial u_{g i}}{\partial t}+u_{g j} \frac{\partial u_{g i}}{\partial x_{j}}-\frac{\partial u_{l i}}{\partial t}-u_{l j} \frac{\partial u_{l i}}{\partial x_{j}}\right) \\
(k=g, l) \\
f_{k i}^{L}=-\Theta_{k} \varepsilon_{i j m}\left(u_{g j}-u_{l j}\right) \omega_{m} \quad(k=g, l)
\end{gathered}
$$

where $\varepsilon_{i j m}$ and $\omega_{m}$ in Eq. [7] are the permutation tensor and the vorticity of the liquid-phase, respectively. The coefficients $\Lambda_{k}, \Gamma_{k}$ and $\Theta_{k}$ in Eqs. [5] [7] are expressed as:

$$
\begin{gathered}
\Lambda_{g}=\frac{1}{8} \frac{a \rho_{l} C_{D}}{\alpha_{g} \rho_{g}}\left|\boldsymbol{u}_{g}-\boldsymbol{u}_{l}\right|, \\
\Lambda_{l}=-\frac{1}{8} \frac{a C_{D}}{\alpha_{l}}\left|\boldsymbol{u}_{g}-\boldsymbol{u}_{l}\right| \\
\Gamma_{g}=\left(\rho_{l} / \rho_{g}\right) C_{V}, \quad \Gamma_{l}=-\left(\alpha_{g} / \alpha_{l}\right) C_{V} \\
\Theta_{g}=\left(\rho_{l} / \rho_{g}\right) C_{L}, \quad \Theta_{l}=-\left(\alpha_{g} / \alpha_{l}\right) C_{L}
\end{gathered}
$$

Here, $a$ is the interfacial area concentration, and $C_{D}, C_{V}$, and $C_{L}$ are the drag coefficient, the virtual mass coefficient, and the lift coefficient, respectively.

The viscous stress $\tau_{k i j}$ is determined by the following equation with reference to the finite element calculations for the two-fluid model presented in the previous papers (Uchiyama \& Minemura, 1997; Uchiyama, 1997, 1999a, 1999b, 2000, 2001).

$$
\tau_{k i j}=\mu_{k}\left(\frac{\partial u_{k i}}{\partial x_{j}}+\frac{\partial u_{k j}}{\partial x_{i}}-\frac{2}{3} \delta_{i j} \frac{\partial u_{k m}}{\partial x_{m}}\right) \quad(k=g, l)
$$

\section{ALE Method}

The computational domain consists of a moving boundary $C_{b}$, which corresponds to the hydrofoil surface, and fixed boundaries $C_{1}, C_{2}$, and $C_{3}$, as shown in Figure 1 . In the ALE finite element method (Uchiyama, 2001), the mesh is rearranged at every computational time step to discretize the domain that involves moving boundary. The rearrangement makes the mesh move with velocity $\hat{\boldsymbol{u}}$. The ALE method calls $\hat{\boldsymbol{u}}$ the mesh velocity. In the case of dividing the 


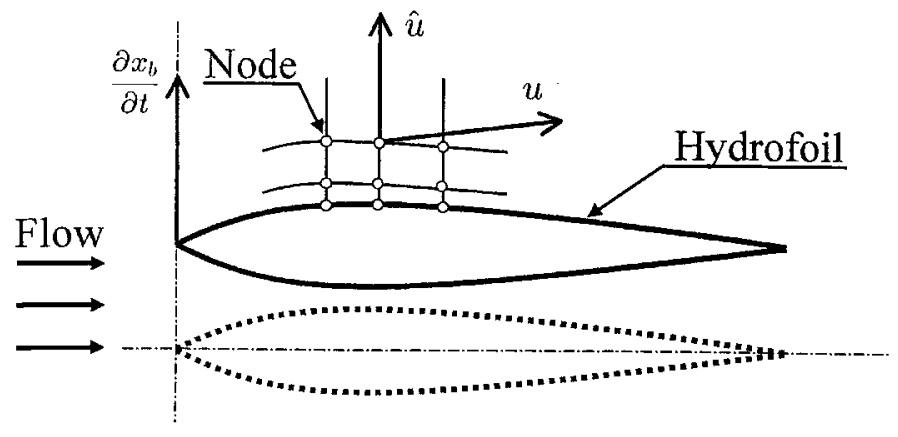

FIGURE 2 Computational mesh and mesh velocity.

flow field into quadrilateral elements as shown in Figure 2, $\hat{\boldsymbol{u}}$ and the fluid velocity $\boldsymbol{u}$ are defined on the nodes.

The time differentials with the spatial coordinate $\boldsymbol{x}$ held constant are involved in Eqs. [2], [3], and [6]. When they are rewritten by considering the mesh velocity $\hat{\boldsymbol{u}}$, the ALE description of the incompressible two-fluid model is obtained (Uchiyama, 2001).

$$
\begin{gathered}
\frac{\partial \alpha_{k}}{\partial t}+\frac{\partial}{\partial x_{j}}\left(\alpha_{k} u_{k j}\right)-\hat{u}_{j} \frac{\partial \alpha_{k}}{\partial x_{j}}=0 \quad(k=g, l) \\
\frac{\partial u_{k i}}{\partial t}+\left(u_{k j}-\hat{u}_{j}\right) \frac{\partial u_{k i}}{\partial x_{j}}=\beta_{k} \Phi_{g i}+\gamma_{k} \Phi_{l i} \quad(k=g, l)
\end{gathered}
$$

where

$$
\begin{aligned}
\Phi_{k i}= & -\frac{1}{\rho_{k}} \frac{\partial p}{\partial x_{i}}+\frac{1}{\alpha_{k} \rho_{k}} \frac{\partial}{\partial x_{j}}\left(\alpha_{k} \tau_{k i j}\right) \\
& +f_{k i}^{D}+f_{k i}^{L} \quad(k=g, l) \\
\left(\begin{array}{cc}
\beta_{g} & \gamma_{g} \\
\beta_{l} & \gamma_{l}
\end{array}\right)= & \left(\begin{array}{cc}
1+\Gamma_{g} & -\Gamma_{g} \\
\Gamma_{l} & 1-\Gamma_{l}
\end{array}\right)^{-1}
\end{aligned}
$$

The boundary condition is listed in Table I. On the inlet boundary $C_{1}$, a uniform flow is imposed and the fluid velocities and the volumetric fractions are given. The fluid traction is assumed to be zero at the outlet $C_{2}$, whereas a slip condition is prescribed on the lateral boundary $C_{3}$. The mesh velocity is zero at these fixed boundaries. On the hydrofoil surface $C_{b}$, a non-slip condition is applied and accordingly the fluid has the same velocity as the hydrofoil.

TABLE I Boundary condition

\begin{tabular}{lll}
\hline & $C_{1}$ & $u_{k i}=\bar{u}_{k i}, \alpha_{k}=\bar{\alpha}_{k}, \hat{u}_{i}=0$ \\
Fixed boundary & $C_{2}$ & $n_{j}\left(\delta_{i j} p-\tau_{k i j}\right)=0, \hat{u}_{i}=0$ \\
& $C_{3}$ & $u_{k 2}=\tau_{k 1}=0, \hat{u}_{i}=0$ \\
Hydrofoil surface & $C_{b}$ & $u_{k 1}=\hat{u}_{1}=0, u_{k 2}=\hat{u}_{2}=\partial x_{b} / \partial t$ \\
\hline
\end{tabular}

\section{Solution Algorithm}

Equations [12] and [13] are solved by the finite element method. The time advancement is based on a fractional step method.

In the first step, an auxiliary velocity $\tilde{u}_{k i}$ and a scalar function $\phi$ are estimated by the following equations:

$$
\begin{gathered}
\tilde{u}_{k i}=b_{k} \Psi_{g i}^{n}+c_{k} \Psi_{l i}^{n} \quad(k=g, l) \\
\frac{\partial}{\partial x_{j}}\left[\left(\frac{\alpha_{g}^{n}}{\rho_{g}^{*}}+\frac{\alpha_{l}^{n}}{\rho_{l}^{*}}\right) \frac{\partial \phi}{\partial x_{j}}\right]=\frac{\partial}{\partial x_{j}}\left(\alpha_{g}^{n} \tilde{u}_{g j}+\alpha_{l}^{n} \tilde{u}_{l j}\right)
\end{gathered}
$$

where

$$
\begin{aligned}
& \Psi_{k i}^{n}=u_{k i}^{n}-\Delta t\left\{\left(u_{k j}^{n}-\hat{u}_{j}^{n}\right) \frac{\partial u_{k i}^{n}}{\partial x_{j}}\right. \\
&-\left[\frac{\beta_{k}^{n}}{\alpha_{g}^{n} \rho_{g}} \frac{\partial}{\partial x_{j}}\left(\alpha_{g}^{n} \tau_{g i j}^{n}\right)+\frac{\gamma_{k}^{n}}{\alpha_{l}^{n} \rho_{l}} \frac{\partial}{\partial x_{j}}\left(\alpha_{l}^{n} \tau_{l j}^{n}\right)\right] \\
&\left.+\left(\beta_{k}^{n} \Theta_{g}^{n}+\gamma_{k}^{n} \Theta_{l}^{n}\right) \varepsilon_{i j m}\left(u_{g j}^{n}-u_{l j}^{n}\right) \omega_{m}^{n}\right\} \quad(k=g, l) \\
& \frac{1}{\rho_{k}^{*}}=b_{k}\left(\frac{\beta_{g}^{n}}{\rho_{g}}+\frac{\gamma_{g}^{n}}{\rho_{l}}\right)+c_{k}\left(\frac{\beta_{l}^{n}}{\rho_{g}}+\frac{\gamma_{l}^{n}}{\rho_{l}}\right) \quad(k=g, l) \\
&\left(\begin{array}{ll}
b_{g} & c_{g} \\
b_{l} & c_{l}
\end{array}\right)=\left(\begin{array}{cc}
1+\Delta t\left(\beta_{g}^{n} \Lambda_{g}^{n}+\gamma_{g}^{n} \Lambda_{l}^{n}\right) & -\Delta t\left(\beta_{g}^{n} \Lambda_{g}^{n}+\gamma_{g}^{n} \Lambda_{l}^{n}\right) \\
\Delta t\left(\beta_{l}^{n} \Lambda_{g}^{n}+\gamma_{l}^{n} \Lambda_{l}^{n}\right) & 1-\Delta t\left(\beta_{l}^{n} \Lambda_{g}^{n}+\gamma_{l}^{n} \Lambda_{l}^{n}\right)
\end{array}\right)^{-1}
\end{aligned}
$$

In the second step, the velocity $u_{k i}^{n+1}$, the pressure $p^{n+1}$, and the volumetric fraction $\alpha_{k}^{n+1}$ are calculated by the following equations:

$$
\begin{gathered}
u_{k i}^{n+1}=\tilde{u}_{k i}-\frac{1}{\rho_{k}^{*}} \frac{\partial \phi}{\partial x_{i}} \quad(k=g, l) \\
p^{n+1}=\phi / \Delta t \\
\alpha_{k}^{n+1}=\alpha_{k}^{n}-\Delta t\left[\frac{\partial}{\partial x_{j}}\left(\alpha_{k}^{n} u_{k j}^{n+1}\right)-\hat{u}_{j}^{n} \frac{\partial \alpha_{k}^{n}}{\partial x_{j}}\right] \\
\quad(k=g, l)
\end{gathered}
$$

\section{Finite Element Equations}

The computational domain is discretized by quadrilateral elements. Figure 3 shows the element. $p$ is defined at the center of the element. $u_{k}, \hat{u}, \alpha_{k}$, and $\phi$ are defined on the vertexes (nodes) of the element. Applying the Galerkin formulation, the finite element equations are expressed as (Uchiyama, 2001):

$$
\begin{gathered}
\boldsymbol{M}^{k} \tilde{\boldsymbol{u}}_{k i}=\boldsymbol{M}_{b}^{k} \boldsymbol{u}_{g i}^{n}+\boldsymbol{M}_{c}^{k} \boldsymbol{u}_{l i}^{n}-\boldsymbol{F}_{k i}^{n} \Delta t \quad(k=g, l) \\
\boldsymbol{T} \boldsymbol{\phi}=-\boldsymbol{R}+\boldsymbol{P} \\
\boldsymbol{M} \boldsymbol{u}_{k i}^{n+1}=\boldsymbol{M} \tilde{\boldsymbol{u}}_{k i}-\boldsymbol{E}_{k i} \quad(k=g, l)
\end{gathered}
$$




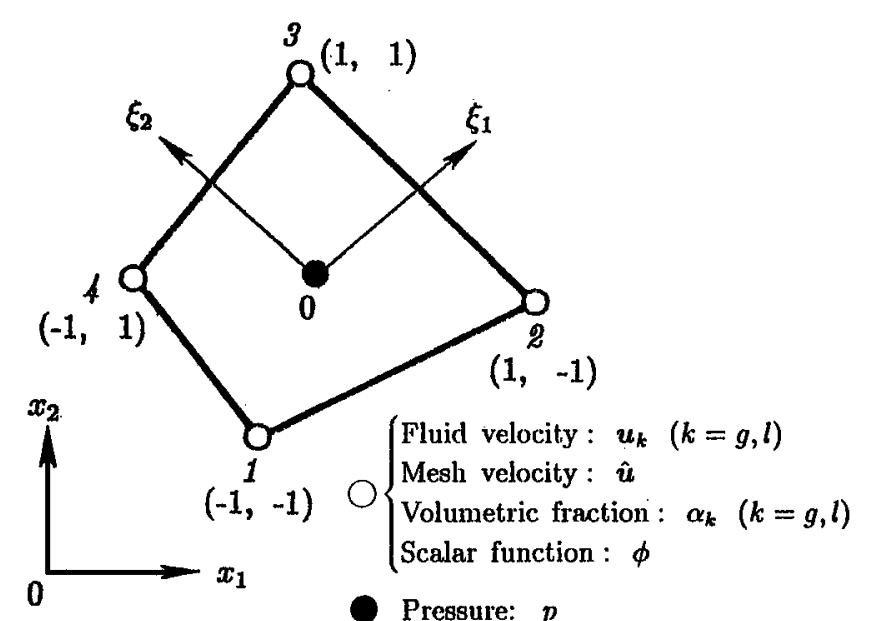

FIGURE 3 Quadrilateral element.

$$
\begin{gathered}
p^{n+1}=S_{\alpha} \phi_{\alpha} /\left(S_{e} \Delta t\right) \\
M \alpha_{k}^{n+1}=\boldsymbol{M} \boldsymbol{\alpha}_{k}^{n}-\left(\boldsymbol{Q}_{k}-C_{k}\right) \Delta t \quad(k=g, l)
\end{gathered}
$$

where

$$
\begin{aligned}
\boldsymbol{M}^{k} & =\sum M_{\alpha \beta}^{k}, \quad \boldsymbol{M}_{b}^{k}=\sum M_{\alpha \beta}^{k} b_{k}, \\
\boldsymbol{M}_{c}^{k} & =\sum M_{\alpha \beta}^{k} c_{k}, \quad \boldsymbol{F}_{k i}^{n}=\sum F_{k i \alpha}^{n}, \\
\boldsymbol{T} & =\sum T_{\alpha \beta \gamma}\left(\frac{\alpha_{g \gamma}^{n}}{\rho_{g}^{*}}+\frac{\alpha_{l \gamma}^{n}}{\rho_{l}^{*}}\right), \\
\boldsymbol{R} & =\sum R_{\alpha \beta j}\left(\alpha_{g \beta}^{n} \tilde{u}_{g j \beta}+\alpha_{l \beta}^{n} \tilde{u}_{l j \beta}\right), \\
\boldsymbol{P} & =\sum P_{\alpha \beta}\left(\frac{\alpha_{g \beta}^{n}}{\rho_{g}^{*}}+\frac{\alpha_{l \beta}^{n}}{\rho_{l}^{*}}\right), \quad \boldsymbol{M}=\sum M_{\alpha \beta}, \\
\boldsymbol{E}_{k i} & =\sum\left(R_{\alpha \beta i} \phi_{\beta} / \rho_{k}^{*}\right), \\
S_{\alpha} & =\int N_{\alpha} d S, \quad \boldsymbol{Q}_{k}=\sum R_{\alpha \beta i} \alpha_{k \beta}^{n} u_{k i \beta}^{n+1}, \\
\boldsymbol{C}_{k} & =\sum C_{\alpha \beta \gamma j} \hat{u}_{j \beta}^{n} \alpha_{k \gamma}^{n}
\end{aligned}
$$

Here, $\sum$ stands for the assembly over the whole domain, and the matrices are defined by the use of a shape function $N$ and a weighting function $W$ (Uchiyama, 1999a, 1999b).

$$
\begin{aligned}
& M_{\alpha \beta}=\int N_{\alpha} N_{\beta} d S, \quad T_{\alpha \beta \gamma}=\int \frac{\partial N_{\alpha}}{\partial x_{j}} \frac{\partial N_{\beta}}{\partial x_{j}} N_{\gamma} d S, \\
& R_{\alpha \beta i}=\int N_{\alpha} \frac{\partial N_{\beta}}{\partial x_{i}} d S, \quad P_{\alpha \beta}=\int N_{\alpha} N_{\beta} \frac{\partial \phi}{\partial x_{i}} n_{i} d C,
\end{aligned}
$$

$$
\begin{aligned}
M_{\alpha \beta}^{k}= & \int W_{\alpha k} N_{\beta} d S, \quad C_{\alpha \beta \gamma j}^{k}=\int W_{\alpha k} N_{\beta} \frac{\partial N_{\gamma}}{\partial x_{j}} d S, \\
C_{\alpha \beta \gamma j}= & \int N_{\alpha} N_{\beta} \frac{\partial N_{\gamma}}{\partial x_{j}} d S, \quad K_{\alpha \beta}=\int \frac{\partial N_{\alpha}}{\partial x_{j}} \frac{\partial N_{\beta}}{\partial x_{j}} d S, \\
K_{\alpha \beta j}^{i}= & \int \frac{\partial N_{\alpha}}{\partial x_{j}} \frac{\partial N_{\beta}}{\partial x_{i}} d S, \quad B_{\alpha \beta}=\int N_{\alpha} N_{\beta} d C, \\
F_{k i \alpha}^{n}= & C_{\alpha \beta \gamma j}^{k}\left[b_{k}\left(u_{g j \beta}^{n}-\hat{u}_{j \beta}^{n}\right) u_{g i \gamma}^{n}+c_{k}\left(u_{l j \beta}^{n}-\hat{u}_{j \beta}^{n}\right) u_{l i \gamma}^{n}\right] \\
& -\left(b_{k} \frac{\beta_{g}^{n}}{\rho_{g}}+c_{k} \frac{\beta_{l}^{n}}{\rho_{g}}\right)\left\{B_{\alpha \beta} \tau_{g i j \beta}^{n} n_{j}\right. \\
& \left.-\mu_{g}\left[K_{\alpha \beta} u_{g i \beta}^{n}+\left(K_{\alpha \beta j}^{n}-\frac{2}{3} K_{\beta \alpha j}^{i}\right) u_{g i \beta}^{n}\right]\right\} \\
& -\left(b_{k} \frac{\gamma_{g}^{n}}{\rho_{l}}+c_{k} \frac{\gamma_{l}^{n}}{\rho_{l}}\right)\left\{B_{\alpha \beta} \tau_{l i j \beta}^{n} n_{j}\right. \\
& \left.-\mu_{l}\left[K_{\alpha \beta} u_{l i \beta}^{n}+\left(K_{\alpha \beta j}^{n}-\frac{2}{3} K_{\beta \alpha j}^{i}\right) u_{l j \beta}^{n}\right]\right\} \\
& +M_{\alpha \beta}\left[b_{k}\left(\beta_{g}^{n} \Theta_{g}^{n}+\gamma_{g}^{n} \Theta_{l}^{n}\right)+c_{k}\left(\beta_{l}^{n} \Theta_{g}^{n}\right.\right. \\
& \left.\left.+\gamma_{l}^{n} \Theta_{l}^{n}\right)\right] \varepsilon_{i j m}\left(u_{g j}^{n}-u_{l j}^{n}\right) \omega_{m}^{n}
\end{aligned}
$$

The weighting function $W_{\alpha k}$ is expressed as (Uchiyama, 1999a, 1999b):

$$
W_{\alpha k}=e^{-a_{k 1}\left(\xi_{1}-\xi_{1 \alpha}\right)-a_{k 2}\left(\xi_{2}-\xi_{2 \alpha}\right)} N_{\alpha} \quad(k=g, l)
$$

where

$$
a_{k 1}=\kappa U_{k \xi_{1}}^{n} /\left|\boldsymbol{U}_{k}^{n}\right|, \quad a_{k 2}=\kappa U_{k \xi_{2}}^{n} /\left|\boldsymbol{U}_{k}^{n}\right| \quad(k=g, l)
$$

In Eq. [26], $\kappa$ is a nonnegative constant, and $U_{k \xi_{1}}^{n}$ and $U_{k \xi_{2}}^{n}$ represent the component of the velocity $\boldsymbol{U}_{k}^{n}$ in the direction of $\xi_{1}$ and $\xi_{2}$, respectively, where $\boldsymbol{U}_{k}^{n}$ is expressed as:

$$
\boldsymbol{U}_{k}^{n}=b_{k} \boldsymbol{u}_{g}^{n}+c_{k} \boldsymbol{u}_{l}^{n} \quad(k=g, l)
$$

The mesh velocity $\hat{\boldsymbol{u}}^{n}$ is determined by Euler's backward scheme based on the mesh arrangements at $t=n \Delta t$ and $(n-1) \Delta t$. The constant $\kappa$ in Eq. [26] is set to be 0.4 in due consideration of prior studies (Uchiyama, 1999a, 1999b). The matrices are estimated by the Gaussian quadrature with $2 \times 2$ Gauss points. The mass matrices $\left(\boldsymbol{M}, \boldsymbol{M}^{k}, \boldsymbol{M}_{b}^{k}\right.$, $\boldsymbol{M}_{c}^{k}$ ) are replaced by the lamped matrices, which are obtained by taking the row sum of the mass matrices and lumping into the diagonal. An LU decomposition method is applied to solve Eq. [20].

\section{NUMERICAL RESULTS AND DISCUSSIONS}

\section{Calculating Condition}

The volumetric fraction of the gas-phase upstream of the hydrofoil, $\alpha_{g 0}$, ranges from 0 to 0.1 , while the Reynolds 
number, defined by the volumetric velocity of the liquidphase $\left(1-\alpha_{g 0}\right) u_{l 0}$ and the chord length $L(=100 \mathrm{~mm})$, is 10000. A bubbly flow is assumed and the bubble diameter is set as $1 \mathrm{~mm}$.

The values of $C_{V}$ and $C_{L}$ are set to be 0.5 and 0.25 , respectively, whereas $C_{D}$ is determined by the following equation (Tomiyama et al., 1995).

$$
C_{D}=\left[\frac{24}{\operatorname{Re}_{b}}\left(1+0.15 R e_{b}^{0.687}\right)+\frac{0.42}{1+42500 R e_{b}^{-1.16}}\right] \alpha_{l}^{-3.5}
$$

where $\operatorname{Re}_{b}=\rho_{l} d\left|\boldsymbol{u}_{g}-\boldsymbol{u}_{l}\right| / \mu_{l}$.

The calculating domain is discretized as illustrated in Figure 4, where the number of elements is $70 \times 57$. A mixing-length turbulent model (Moore \& Moore, 1985)

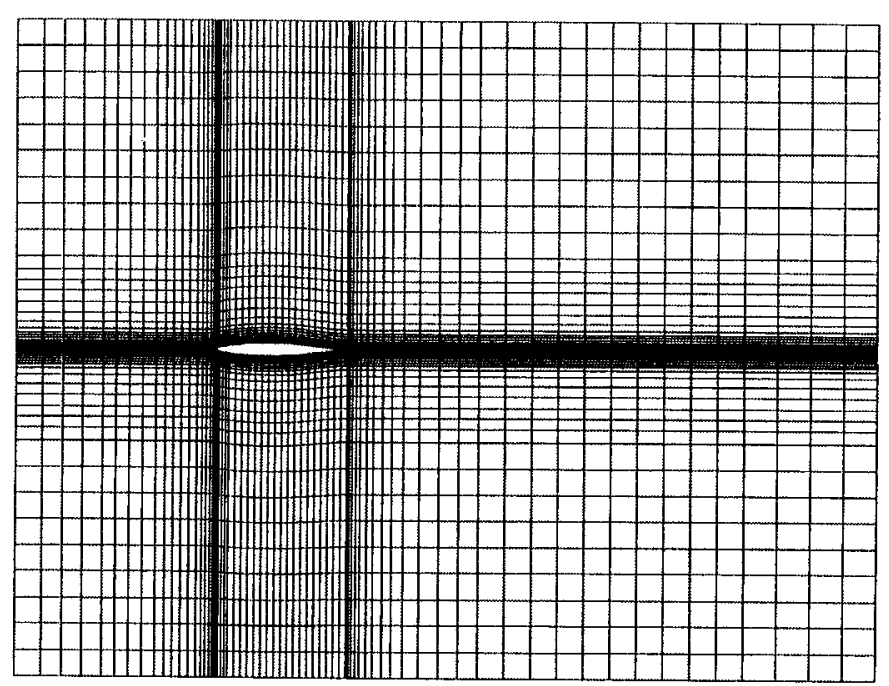

FIGURE 4 Discretization of computational domain is applied to the liquid-phase. The initial condition is given by the flow field for the stationary hydrofoil.

\section{Numerical Results}

When the force acting on the hydrofoil $\boldsymbol{F}$ is estimated from the stress distribution on the stationary hydrofoil, the components of the dimensionless force $\boldsymbol{F} /\left(\rho_{l} u_{l 0}^{2} L / 2\right)$ in the $x_{1}$ - and $x_{2}$-directions, $F_{1}$ and $F_{2}$, respectively, change as functions of the dimensionless time as shown in Figure 5. $F_{1}$ and $F_{2}$ correspond to the drag and lift forces, respectively. The $\alpha_{g 0}$ value scarcely affects the forces. $F_{1}$ remains almost unaltered, but $F_{2}$ fluctuates with small amplitude.

Figure 6 shows the time variations of the forces $F_{1}$ and $F_{2}$ acting on the oscillating hydrofoil, where the results for $\alpha_{g 0}=0.05, a / L=0.05, \omega=4$ are plotted by solid lines. The dimensionless displacement of the hydrofoil $x_{b} / L$ is also indicated at the top of the figure. The force $F_{1}$ changes sinuously, and the period is half of the oscillation period of the hydrofoil. $F_{1}$ takes its maximum value when the hydrofoil is at a standstill, and reaches its minimum value when the hydrofoil passes through the center of the oscillation with its maximum speed. $F_{1}$ is lower than zero except at the maximum value. This suggests that thrust force acts when the hydrofoil is in motion, while drag force acts when it is at rest. The force $F_{2}$, corresponding to the lift force, also changes sinuously, and it has the same period as the hydrofoil oscillation. The forces $F_{1}$ and $F_{2}$ under liquid single-phase flow condition $\left(\alpha_{g 0}=0\right)$ are also plotted using broken lines. The thrust $\left(F_{1}\right)$ and the lift $\left(F_{2}\right)$ diminish under two-phase flow condition. But their periods are not affected by $\alpha_{g 0}$.

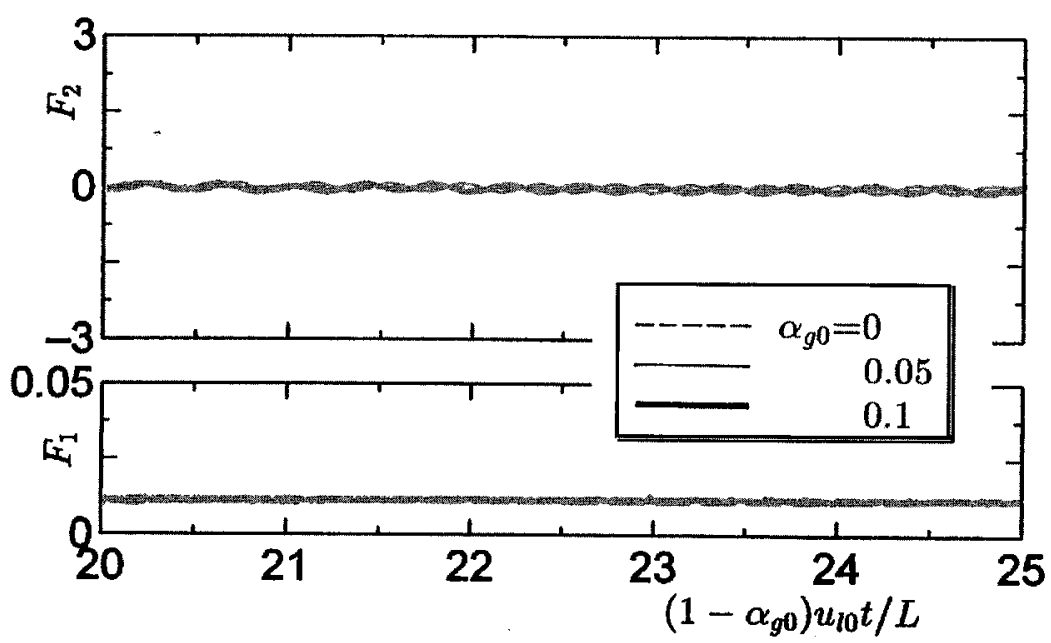

FIGURE 5 Time variation of fluid forces on stationary hydrofoil. 

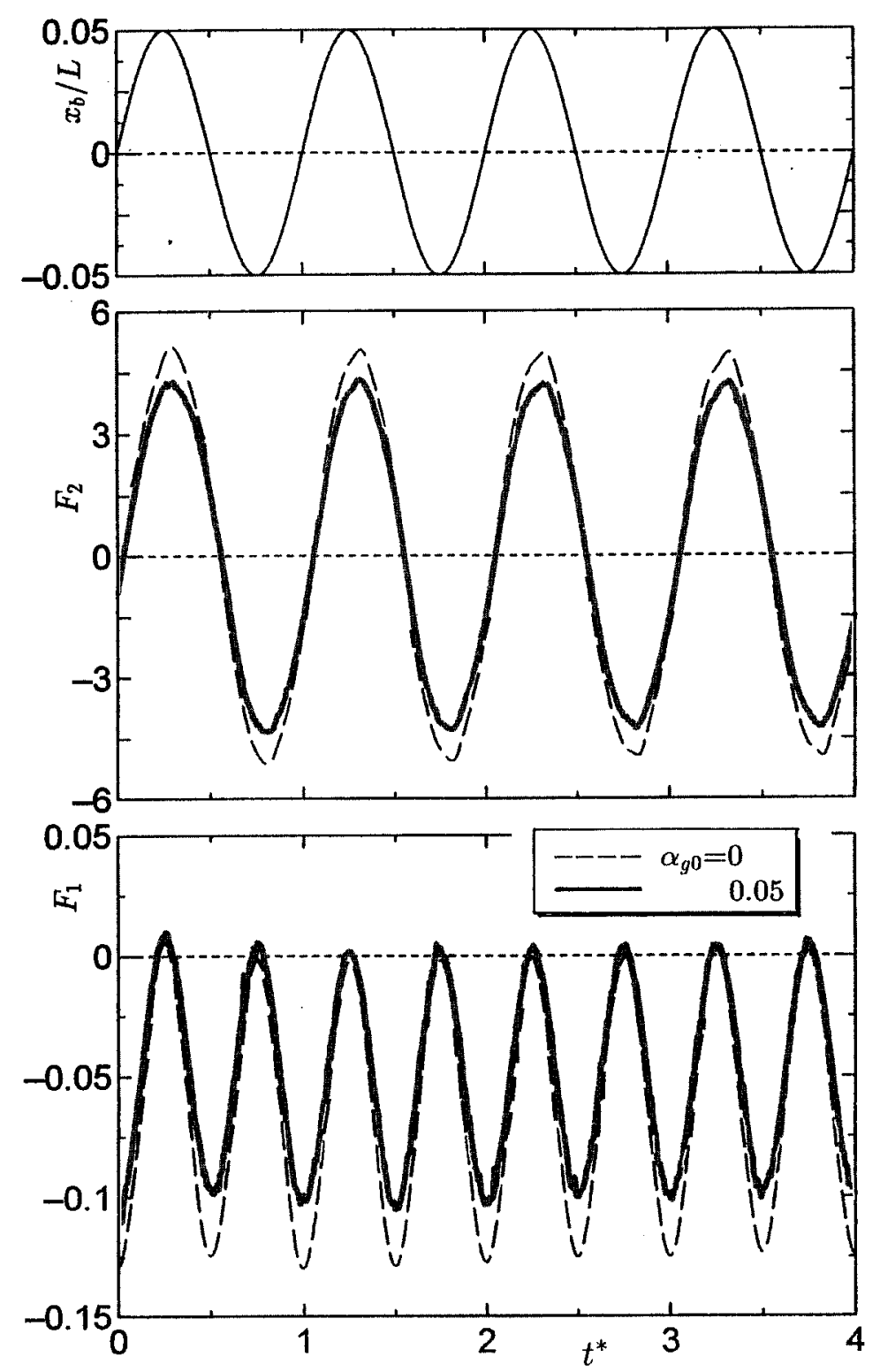

FIGURE 6 Time variation of fluid forces on oscillating hydrofoil $(\omega=4, a / L=0.05)$.

When the $\omega$ value is decreased from 4 to 2 at the same oscillation amplitude as in Figure 6, the forces $F_{1}$ and $F_{2}$ change, as shown in Figure 7. The forces show sinuous change. In comparison with the result at $\omega=4$ indicated in Figure 6, the thrust force decreases and the drag force increases, as seen from the change in $F_{1}$, and the lift force lessens as seen from $F_{2}$. This is because the energy given to the two-phase mixture lowers due to the decrement in the oscillating speed of the hydrofoil.

The change in the time-averaged value of $F_{1}, \overline{F_{1}}$, against $\alpha_{g 0}$ is shown in Figure 8, where the results at $\omega=2$ and 4 are plotted. The absolute value of $\overline{F_{1}}$ decreases with an increment in $\alpha_{g 0}$. The result of $\overline{F_{1}}=0$ is calculated when $\omega=2$ and $\alpha_{g 0}=0.1$, indicating that no thrust force acts on the hydrofoil.

When the absolute value of the lift force $F_{2},\left|F_{2}\right|$, is calculated, the time-averaged value $\overline{\left|F_{2}\right|}$ changes as functions of $\alpha_{g 0}$, as shown in Figure 9. The $\overline{\left|F_{2}\right|}$ value also lessens linearly with the increment in $\alpha_{g 0}$.

Figure 10 shows the Lissajous figures of the vector $\left(F_{1}, F_{2}\right)$ in the case of $a / L=0.05$ and $\omega=4$. The locus of $\left(F_{1}, F_{2}\right)$ produces a figure-eight irrespective of $\alpha_{g 0}$. With the increment in $\alpha_{g 0}$, the thrust and lift forces lessen and the Lissajous figure becomes smaller. This demonstrates that the decrement in the thrust force due to $\alpha_{g 0}$ is almost the same as that in the lift force. It should also be mentioned 

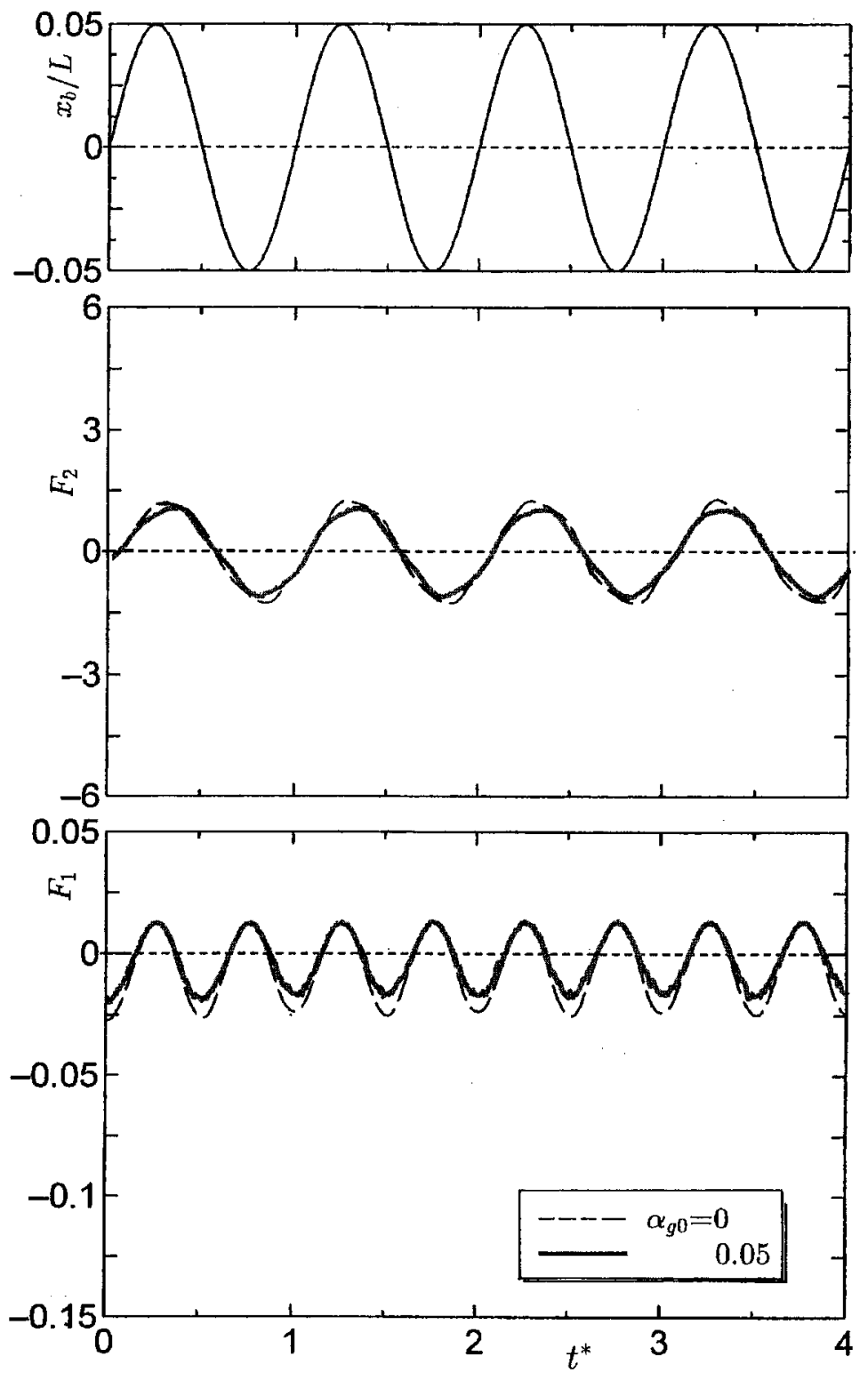

FIGURE 7 Time variation of fluid forces on oscillating hydrofoil $(\omega=2, a / L=0.05)$.

that the Lissajous figure is not smooth but exhibits sawtoothed curves under the two-phase flow conditions. This is attributable to the fluctuations of the flow properties near the hydrofoil. The existence of such fluctuations are indeed confirmed from the changes in $F_{1}$ and $F_{2}$, as shown in Figures 6 and 7. Similar fluctuations are also observed in the calculation of the two-phase flow around a fixed cylinder (Uchiyama, 1999b).

The time variations of the flow properties around the oscillating hydrofoil are shown in Figures 11 through 13, where $\alpha_{g 0}=0.05$ and $a / L=0.05$. The instantaneous distributions at five time points during a half period $\left(3 \leq t^{*} \leq 3.5\right)$ are depicted. The arrows on the left of each figure indicate the instantaneous velocity of the hydrofoil.

The change in the volumetric velocity of the liquidphase, $\alpha_{\mathcal{L}} u_{\text {l }}$, is shown in Figure 11. To illustrate the vortices more definitely, the velocity upstream of the hydrofoil $\alpha_{10} u_{l 0}$ is subtracted. When the hydrofoil passes upward through the oscillation center with its maximum speed (Figure 11(a)), a clockwise vortex shed from the hydrofoil accelerates the flow behind the trailing edge of the hydrofoil. A jet is produced behind the hydrofoil by the oscillation, and its reaction yields the thrust force. The increment in $\alpha_{g 0}$ diminishes the thrust force as shown in 


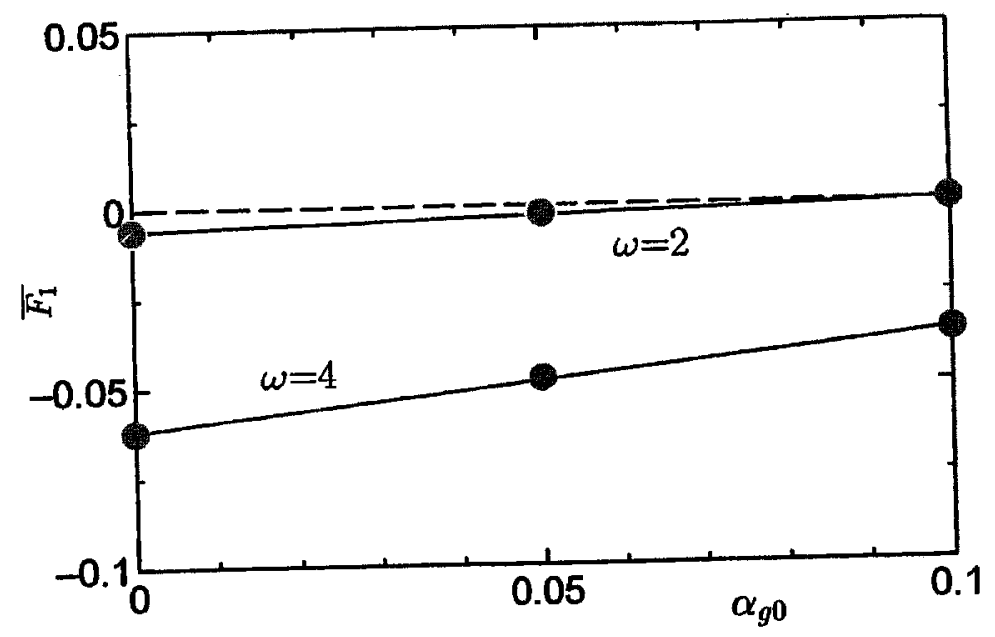

FIGURE 8 Change in thrust force due to $\alpha_{g 0}$.

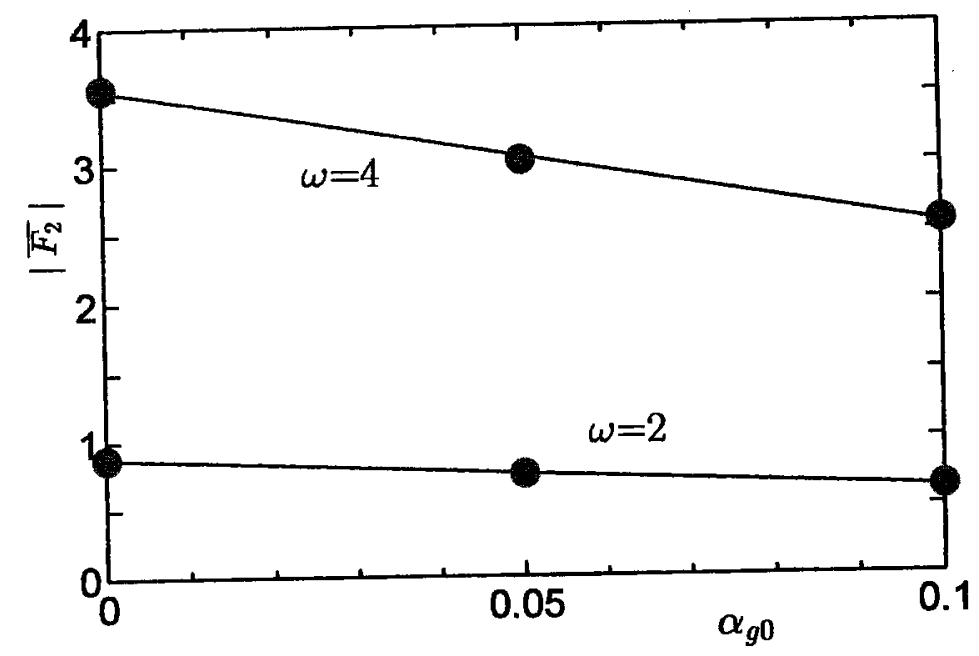

FIGURE 9 Change in lift force due to $\alpha_{g 0}$.

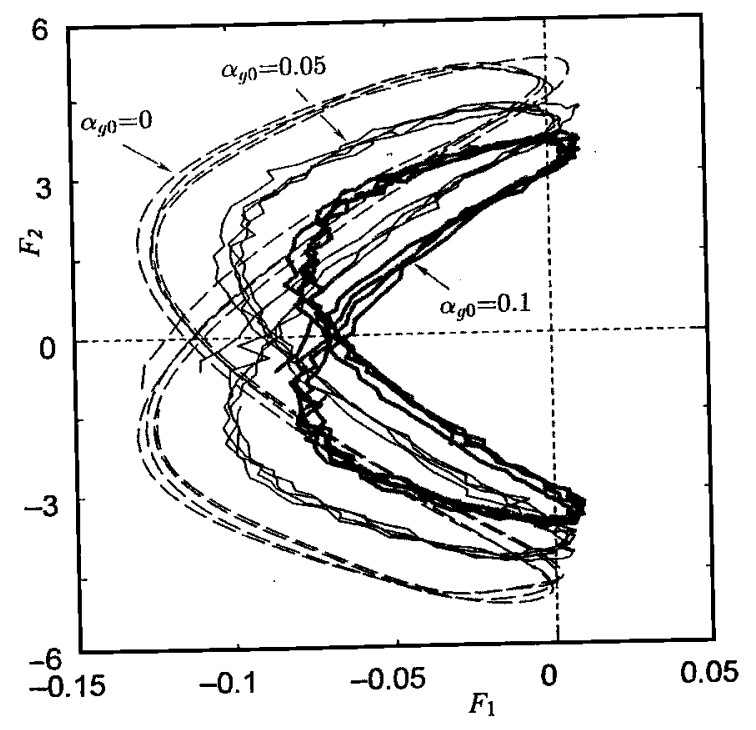

FIGURE 10 Lissajous figure of $\left(F_{1}, F_{2}\right)$ for $\omega=4$ and $a / L=0.05$.
Figures 4 through 8 and 10 . This is because the momentum of the jet lessens owing to the decrement in the fluid density. When the hydrofoil is decelerated (Figure 11(b)) and at rest (Figure $11(\mathrm{c})$ ), the vortex moves away while its strength decreases. When the hydrofoil is accelerated (Figure 11(d)), a counter-clockwise vortex is shed and it accelerates the flow. Figure 11(e) is the periodic flow field of Figure 11(a).

Figure 12 shows the variation of the pressure coefficient $C_{p}$. When the hydrofoil is at its highest speed (Figure 12(a)), the pressure takes its minimum value at the center of the vortex behind the hydrofoil. When the hydrofoil is decelerated (Figure 12(b)), the pressure lowers on the upper surface and heightens on the lower surface. When the hydrofoil is at rest (Figure 12(c)), the pressure difference between the upper and lower sides of the hydrofoil becomes larger. Such periodical change in the pressure distribution causes variation of the lift force, as shown in Figure 6. 


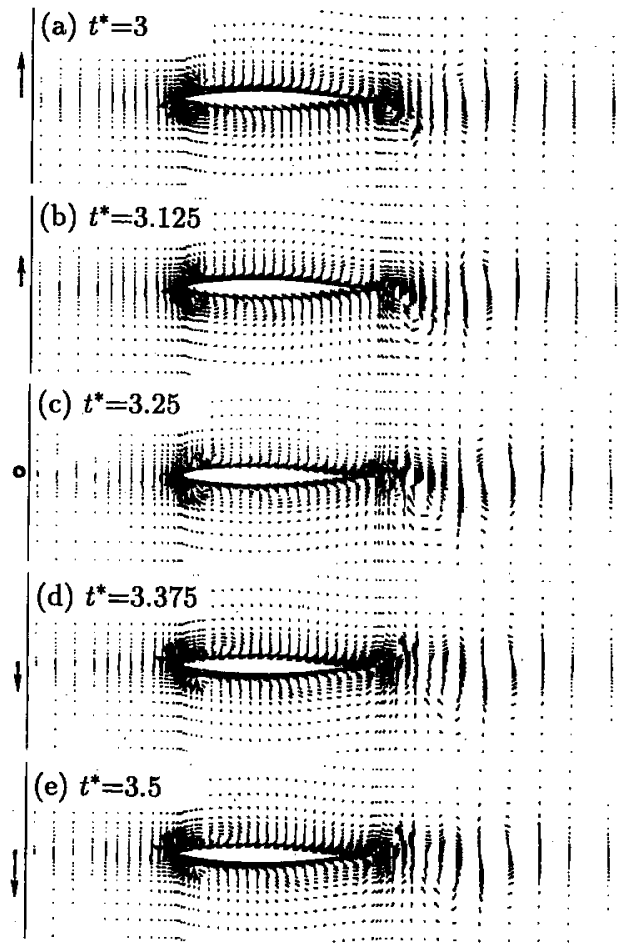

FIGURE 11 Distribution of volumetric velocity of liquid-phase $\alpha_{l} u_{t}$ $\left(\alpha_{g 0}=0.05, \omega=4, a / L=0.05\right)$.
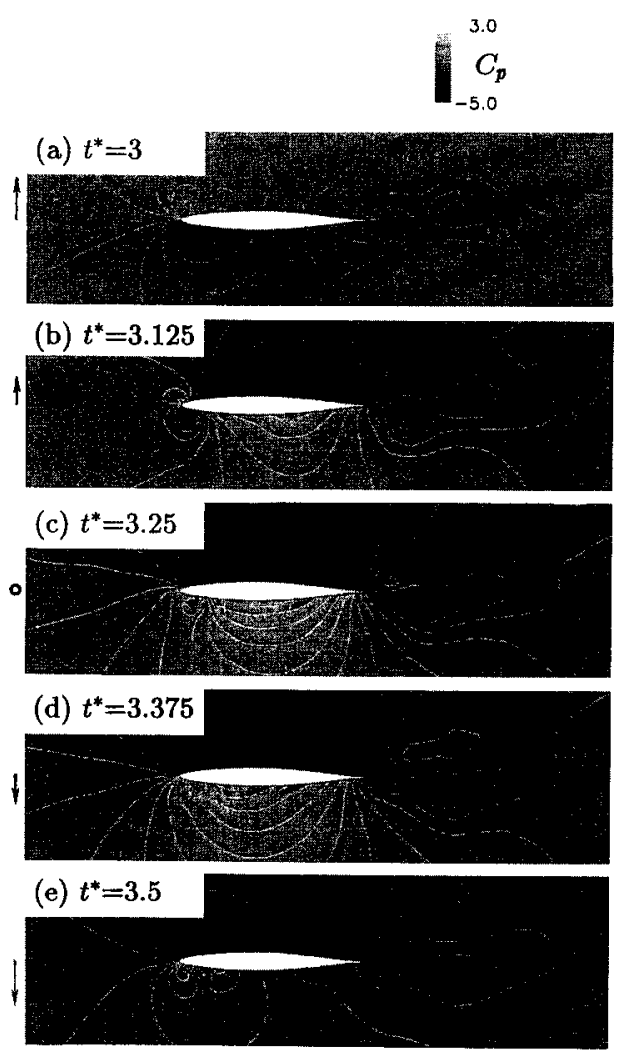

FIGURE 12 Distribution of pressure coefficient $C_{p}\left(\alpha_{g 0}=0.05, \omega=4\right.$, $a / L=0.05)$

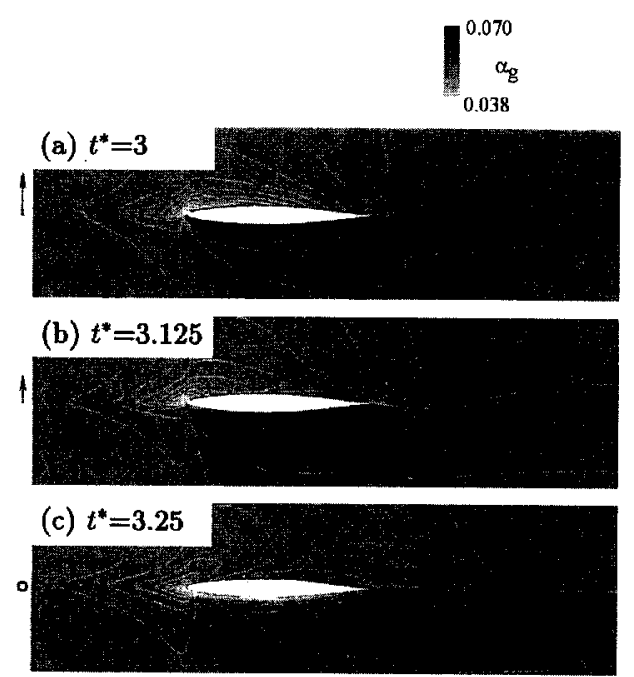

(d) $t^{*}=3.375$

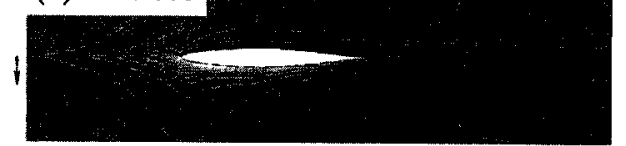

(e) $t^{*}=3.5$

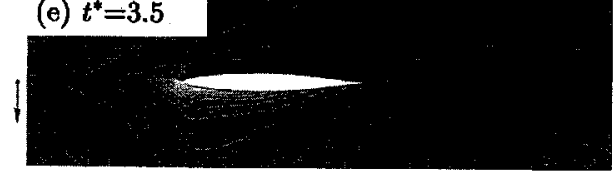

FIGURE 13 Distribution of volumetric fraction of gas-phase $\alpha_{g}$ $\left(\alpha_{g 0}=0.05, \omega=4, a / L=0.05\right)$.

The time variation of the volumetric fraction of the gas-phase $\alpha_{g}$ is shown in Figure 13. When the hydrofoil is at its highest speed (Figure 13(a)), $\alpha_{g}$ is higher on the lower surface and lower on the upper surface, while $\alpha_{g}$ is also higher in the vortex behind the hydrofoil. When the hydrofoil is decelerated (Figure 13(b)) and at rest (Figure 13(c)), the uneven distribution of $\alpha_{g}$ becomes less remarkable. When the hydrofoil is accelerated (Figure 13(d)), the $\alpha_{g}$ value increases on the upper surface.

\section{SUMMARY}

The bubbly flow around a hydrofoil (NACA65-010), which is forced to oscillate normal to the uniform flow, was calculated by an incompressible two-fluid model with the use of the ALE finite element method. The numerical results are summarized as follows:

(1) The thrust and lift forces acting on the hydrofoil change periodically in accordance with the oscillation of the hydrofoil. The forces decrease with an increment in the volumetric fraction of the gas-phase upstream of the hydrofoil $\alpha_{g 0}$. 
(2) The decrement in the thrust force due to $\alpha_{g 0}$ is almost the same as that in the lift force.

(3) The vortex, shed from the trailing edge of the hydrofoil, accelerates the flow behind the hydrofoil, and its reaction yields the thrust force on the hydrofoil.

(4) The flow field around the hydrofoil varies periodically owing to the oscillation of the hydrofoil, resulting in the periodical change in the thrust and lift forces.

\section{NOMENCLATURE}

a oscillation amplitude

$C$ boundary of $S$

$C_{p}$ pressure coefficient $=\left(p-p_{0}\right) /\left(\rho_{l} u_{l 0}^{2} / 2\right)$

$F$ force acting on hydrofoil

$L$ chord length

$n$ unit vector normal to boundary

$p$ pressure

$S$ computational domain

$S_{e}$ area of element

$t$ time

$T$ oscillation period

u velocity

$\hat{\boldsymbol{u}}$ mesh velocity

$\boldsymbol{x}$ orthogonal coordinates

$x_{b} \quad$ displacement of hydrofoil

$\alpha$ volumetric fraction

$\mu$ viscosity

$\xi$ local coordinates

$\rho$ density

$\tau$ viscous stress

$\omega \quad$ dimensionless frequency $=\pi L /\left(1-\alpha_{g 0}\right) u_{l 0} T$

\section{Subscripts}

$\begin{array}{ll}0 & \text { uniform flow } \\ g & \text { gas-phase } \\ l & \text { liquid-phase }\end{array}$

\section{REFERENCES}

Ichikawa, Y. et al. (1991) Bubble Motion Around an Airfoil in Bubbly Flow, Proc. Cavitation and Multiphase Flow Forum, ASME, pp. $155-158$.

Ishii, M. (1975) Thermo-Fluid Dynamic Theory of Two-Phase Flow, Eyrolles, Paris.

Kastner, W. and Seeberger, G. J. (1983) Pump Behavior and Its Impact on A Loss-of-Coolant Accident in a Pressurized Water Reactor, Nucl. Tech., 60, 268-277.

Mikielewicz, J. et al. (1978) A Method for Correlating the Characteristics of Centrifugal Pumps in Two-Phase Flow, Trans. ASME J. Fluid Eng., 100(4), 395-409.

Minemura, K. and Uchiyama, T. (1993) Three-Dimensional Calculation of Air-Water Two-Phase Flow in Centrifugal Impeller Based on a Bubbly Flow Model, Trans. ASME, J. Fluid Eng., 115(4), 766-771.

Moore, J. and Moore, J. G. (1985) Performance Evaluation of Linear Turbine Cascades Using Three-Dimensional Viscous Flow Calculations, Trans. ASME J. Eng. Gas Turbine Power, 107(4), 969-975.

Narabayashi, T. et al. (1986) Centrifugal Pump Behavior in Steady and Transient Two-Phase Flow, J. Nucl. Sci. Technol., 23(2), 136-150.

Nishikawa, H. et al. (1991) The Numerical Calculation of the Bubbly Two Phase Flow Around an Airfoil, Computers and Fluids, 19, $453-460$.

Ohashi, H. and Ishikawa, N. (1971) Flow Visualization around Trailing Edge of Unsteady Airfoil, J. JSME, 74(634), 1500-1507.

Oshima, Y. (1982) Experimental Study of the Vortical Flow Behind an Oscillating Airfoil, J. Jap. Soci. Fluid Mech., 1, 67-79.

Shida, Y. et al. (1986) New Computational Method for a Flow of a Bubbly Liquid, Proc. Cavitation and Multiphase Flow Forum, ASME, pp. $46-49$.

Tomiyama, A. et al. (1995) A Simple Numerical Method for Solving an Incompressible Two-Fluid Model in a General Curvilinear Coordinate System, Proc. 2nd Int. Conf. Multiphase Flow, pp. NU23 - NU30.

Uchiyama, T. and Minemura, K. (1997) Finite Element Method for Gas Liquid Two-Phase Flow Based on an Incompressible Two-Fluid Model, Comput. Model. Simulat. Eng., 2, 23-37.

Uchiyama, T. (1997) Numerical Analysis of an Incompressible Two-Fluid Model by Finite Element Method Based on a Fractional Step Method, Comput. Model. Simulat. Eng., 2, 363-385.

Uchiyama, T. (1999a) Petrov-Galerkin Finite Element Method for Gas-Liquid Two-Phase Flow Based on an Incompressible Two-Fluid Model, Nucl. Eng. Des., 193, 145-157.

Uchiyama, T. (1999b) Numerical Simulation of Gas-Liquid Two-Phase Flow Around a Rectangular Cylinder by the Incompressible Two-Fluid Model, Nucl. Sci. Eng., 133, 92-105.

Uchiyama, T. (2000) Numerical Analysis of Air-Water Two-Phase Flow Across a Staggered Tube Bundle Using an Incompressible Two-Fluid Model, Nucl. Sci. Eng., 134, $281-292$.

Uchiyama, T. (2001) ALE Finite Element Method for Gas-Liquid TwoPhase Flow Including Moving Boundary Based on an Incompressible Two-Fluid Model, Nucl. Eng. Des., 205, 69-82. 

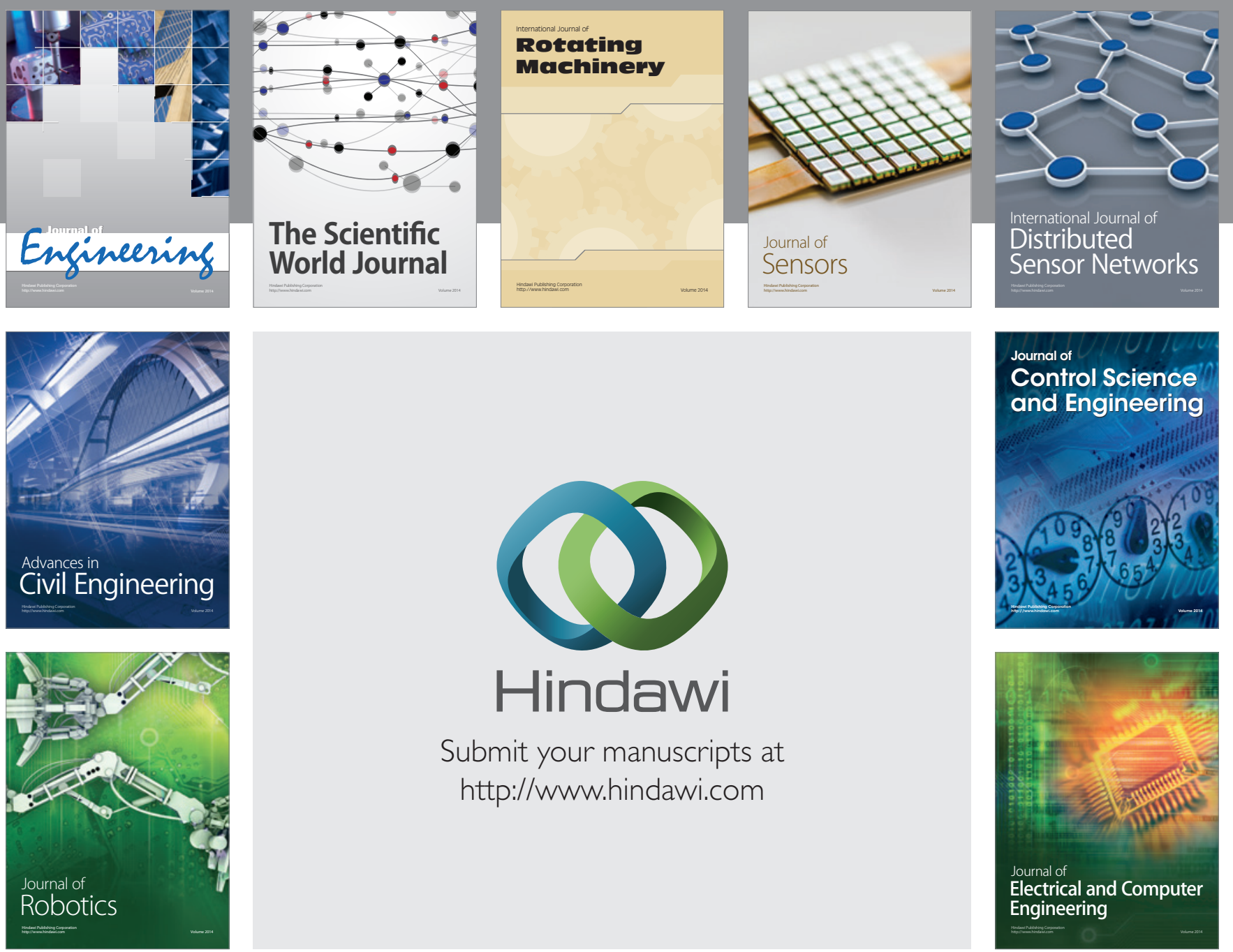

Submit your manuscripts at

http://www.hindawi.com
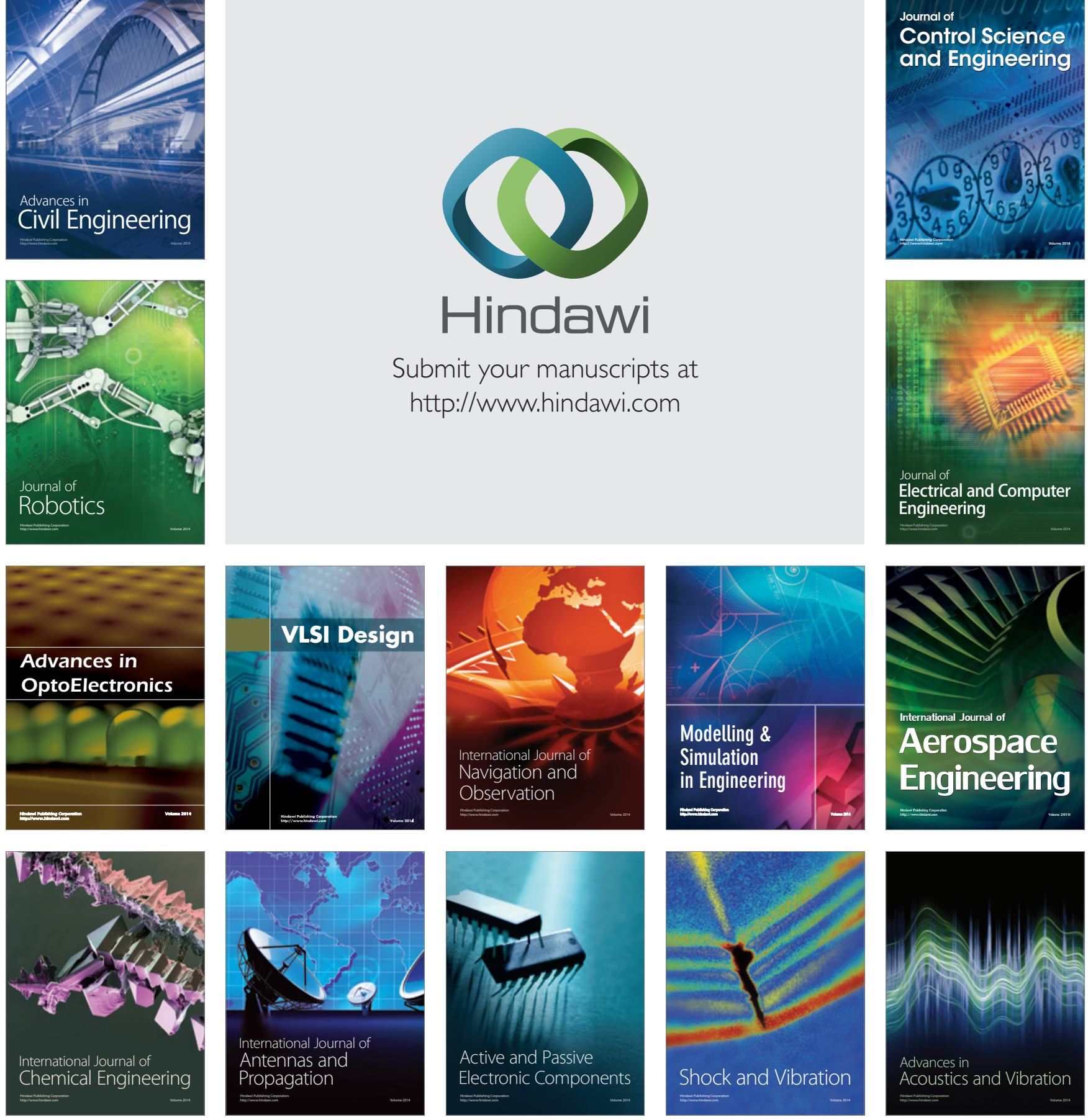